\title{
Clinical implications for patients treated inappropriately for community-acquired pneumonia in the emergency department
}

\author{
Scott T Micek', Adam Lang ${ }^{2}$, Brian M Fuller ${ }^{3}$, Nicholas B Hampton ${ }^{4}$ and Marin H Kollef ${ }^{*}$
}

\begin{abstract}
Background: Community-acquired pneumonia (CAP) is one of the most common infections presenting to the emergency department (ED). Increasingly, antibiotic resistant bacteria have been identified as causative pathogens in patients treated for CAP, especially in patients with healthcare exposure risk factors.

Methods: We retrospectively identified adult subjects treated for CAP in the ED requiring hospital admission (January 2003-December 2011). Inappropriate antibiotic treatment, defined as an antibiotic regimen that lacked in vitro activity against the isolated pathogen, served as the primary end point. Information regarding demographics, severity of illness, comorbidities, and antibiotic treatment was recorded. Logistic regression was used to determine factors independently associated with inappropriate treatment.
\end{abstract}

Results: The initial cohort included 259 patients, 72 (27.8\%) receiving inappropriate antibiotic treatment. There was no difference in hospital mortality between patients receiving inappropriate and appropriate treatment ( $8.3 \%$ vs. $7.0 \%$; $p=0.702)$. Hospital length of stay ( $10.3 \pm 12.0$ days vs. $7.0 \pm 8.9$ days; $p=0.017)$ and 30 -day readmission $(23.6 \%$ vs. $12.3 \% ; p=0.024)$ were greater among patients receiving inappropriate treatment. Three variables were independently associated with inappropriate treatment: admission from long-term care (AOR, 9.05; 95\% Cl, 3.93-20.84), antibiotic exposure in the previous 30 days (AOR, 1.85; $95 \% \mathrm{Cl}, 1.35-2.52)$, and chronic obstructive pulmonary disease (AOR, 2.05; $95 \% \mathrm{Cl}, 1.52-2.78)$.

Conclusion: Inappropriate antibiotic treatment of presumed CAP in the ED negatively impacts patient outcome and readmission rate. Knowledge of risk factors associated with inappropriate antibiotic treatment of presumed CAP could advance the management of patients with pneumonia presenting to the ED and potentially improve patient outcomes.

Keywords: Pneumonia, Antibiotics, Resistant pathogens, Outcomes

\section{Background}

Community-acquired pneumonia (CAP) is an important infection globally, and accounts for significant morbidity, mortality, and economic burden [1-5]. Mortality may be as high as $14 \%$ overall, whereas for the elderly, mortality reaches greater than $50 \%$ within 5 years $[1,5]$. Treatment of CAP is potentially compromised by the rapid emergence of bacterial resistance to the most commonly prescribed antibiotics, including beta-lactams, macrolides, and fluoroquinolones [6]. Even multidrug-resistant

\footnotetext{
* Correspondence: mkollef@dom.wustl.edu

${ }^{5}$ Division of Pulmonary and Critical Care Medicine, Washington University School of Medicine, 660 South Euclid Avenue, Campus Box 8052, St. Louis, MO 63110, USA

Full list of author information is available at the end of the article
}

(MDR) strains (commonly defined as resistance to three or more of the commonly used classes of agents) are emerging [6]. Traditionally, pneumonia developing in recently hospitalized patients or those residing in longterm care facilities, nursing homes, or undergoing dialysis, has been classified and treated as CAP. However, a new pneumonia classification, distinct from CAP - healthcareassociated pneumonia (HCAP) - has been introduced to address patients outside of the hospital at risk of infection with MDR pathogens [7]. The 2005 American Thoracic Society/Infectious Disease Society of America (ATS/IDSA) guidelines also recognized HCAP as a new category of pneumonia [8]. 
Patients with HCAP risk factors are at increased risk of infection with MDR pathogens [9-16]. Physicians should be aware that microbiological features and clinical outcomes of patients with HCAP are more similar to patients with hospital-acquired pneumonia (HAP) than those with traditional CAP [9]. A critical disparity appears to be the more frequent administration of inappropriate antibiotic treatment in patients with HCAP compared to those with CAP, secondary to a higher incidence of antibiotic-resistant pathogens in HCAP [13]. However, factors associated with inappropriate antibiotic administration in the emergency department (ED), and the short- and long-term impact of inappropriate therapy has not been well studied. Therefore, we performed an investigation to assess the incidence of inappropriate initial antibiotic treatment of hospitalized patients with presumed CAP presenting to the ED. We also aimed to assess risk factors associated with inappropriate antibiotic treatment and its relationship to other clinical outcomes.

\section{Methods}

Study overview and subjects

We retrospectively identified all hospitalized adult (age $>18$ years) patients diagnosed and treated for culture-positive CAP in the ED between January 1, 2003 and December 31, 2011. Presumed CAP was defined as a new or progressive radiographic infiltrate plus one of the following: temperature greater than $38.3^{\circ} \mathrm{C}$, WBC greater than $10,000 / \mu \mathrm{L}$, or purulent tracheal secretions. One investigator (MHK), blinded to the clinical and microbiologic information adjudicated the chest imaging. To be included, patients had to be treated in the ED with a CAP antimicrobial regimen that included at our institution ceftriaxone plus azithromycin or moxifloxacin. Identification of pathogens was based on the results of cultures obtained in the ED from blood, pleural fluid, sputum, or the lower airways. A positive urinary antigen for Legionella pneumophilia serogroup 1 was used to document infection with this pathogen. The Washington University School of Medicine Human Studies Committee approved the study.

\section{Endpoints and covariates}

Patients receiving inappropriate therapy, defined as an antibiotic regimen that lacked in vitro activity against the isolated pathogen, were compared to those receiving appropriate antibiotic therapy. Covariates of interest included patient demographics, severity of illness, the Charlson score, and antibiotic treatment. Demographic factors included age, gender, and race. Comorbidities potentially influencing the isolated pathogen included residence in a long-term care facility, hospitalization in the last 90 days, receipt of antimicrobials in the last 30 days, end-stage renal disease requiring hemodialysis, and immunosuppression. Immunosuppression was defined as the presence of the human immunodeficiency virus (HIV), active malignancy undergoing chemotherapy, or treatment with immunosuppressants (i.e., $10 \mathrm{mg}$ prednisone or equivalent daily for at least 30 days or alternate agents). Disease severity was assessed with the CURB-65 score, along with the need for either intensive care unit (ICU) admission or mechanical ventilation [17]. Infectionrelated variables included the presence of bacteremia complicating the pneumonia, and the presence of polymicrobial infection. We classified the initial antibiotic regimen as appropriate if the antibiotic regimen demonstrated in vitro activity against the pathogen(s) isolated and was administered within 6 hours of presentation.

\section{Statistics}

We completed univariate analyses with either the Fisher's exact test or Student's t-test as appropriate. Continuous, non-parametrically distributed data was compared via the Mann-Whitney U test. All analyses were two tailed, and a $p$ value of $<0.05$ was statistically significant. Logistic regression was used to determine independent factors associated with inappropriate treatment. Variables significant at $\mathrm{p}<0.10$ in univariate analyses were entered into the model. To arrive at the most parsimonious model we utilized a step-wise backward elimination approach. Co-linearity was explored with correlation matrices. Adjusted odds ratios (AORs) and ninety-five percent confidence intervals (CIs) are reported where appropriate. The model's goodness-offit was assessed via calculation of the $\mathrm{R} 2$ value and the Hosmer-Lemeshow c-statistic. All analyses were performed with SPSS 11.0 (SPSS, Chicago, IL).

\section{Results}

During the study period there were 1367 patients with culture-positive bacterial pneumonia admitted from the ED. Among these 1108 (81.5\%) were diagnosed and treated for presumed HCAP, received initial treatment with broad-spectrum antibiotics and excluded from analysis. Of the 259 patients diagnosed and treated for CAP, $187(72.2 \%)$ received appropriate antibiotic treatment and $72(27.8 \%)$ received inappropriate treatment (Figure 1). Overall hospital mortality was $7.3 \%$ and 30 -day hospital readmission was $15.4 \%$. The average hospital length of stay was 7.9 days (median 5 days).

Table 1 shows the characteristics of patients receiving appropriate and inappropriate antibiotic treatment. There were no differences in demographics or severity of illness between groups. There was a trend towards greater ICU admission for patients receiving inappropriate treatment $(\mathrm{p}=0.052)$.

With respect to infection-related characteristics, patients receiving appropriate treatment were more likely 


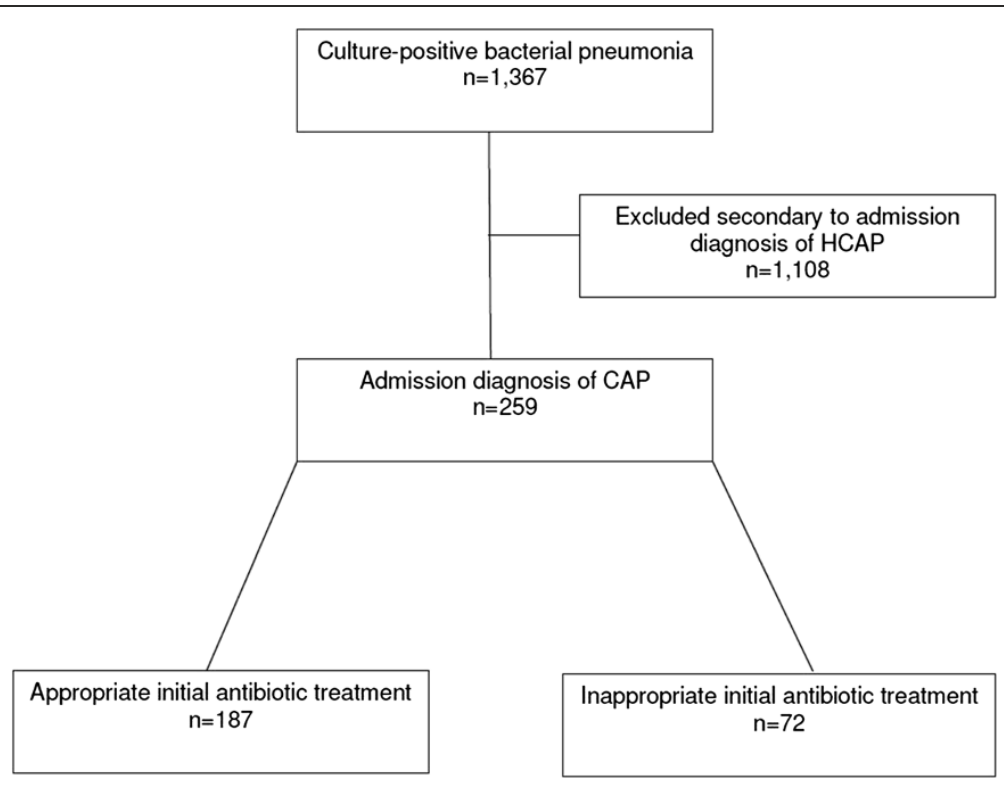

Figure 1 Flow diagram depicting bacterial pneumonia patients admitted from the emergency department. HCAP: healthcare-associated pneumonia; CAP: community-acquired pneumonia.

to be infected with Streptococcus pneumoniae, MSSA, Escherichia coli and Haemophilus influenzae (Table 2). MRSA, Pseudomonas aeruginosa, Enterobacter species and Acinetobacter species were more common among patients receiving inappropriate treatment. MRSA was the most common pathogen accounting for inappropriate treatment (47.2\%) followed by Pseudomonas aeruginosa (27.8\%). Tracheal aspirates and bronchoalveolar lavage specimens were more often used to establish the microbiologic diagnosis among patients receiving inappropriate treatment. There was no difference in the presence of polymicrobial infection or secondary bacteremia between groups.

We observed an association between comorbidities and inappropriate treatment. The median Charlson score was higher in patients receiving inappropriate treatment ( 5 vs. $4, \mathrm{p}=0.090$ ). Neurologic conditions such as dementia and stroke occurred equally between groups while other chronic diseases, such as chronic obstructive pulmonary disease (COPD), chronic kidney disease, and peripheral vascular disease occurred more often in patients receiving inappropriate treatment. Of the comorbidities evaluated, those linked to the definition of HCAP correlated with inappropriate treatment (Table 1). For example, long-term care patients were more than nine times (OR, 9.96; 95\% CI, 2.02-49.18) as likely to receive inappropriate treatment. Patients on chronic dialysis (OR, 5.58; 95\% CI, 1.36-22.93) or having received previous antibiotic treatment (OR, 2.12; 95\% CI, 1.18-3.79) were also significantly more likely to receive inappropriate treatment.

Overall, 129 (49.8\%) patients were retrospectively found to have at least one HCAP risk factor. Patients having any of the HCAP risk factors were significantly less likely to receive appropriate antibiotic treatment compared to patients with no HCAP risk factors $(61.2 \%$ versus $83.1 \% ; \mathrm{p}<0.001)$. Inappropriate antibiotic treatment was not associated with excess mortality (Table 3). However, hospital length of stay was significantly longer among patients receiving inappropriate treatment. Similarly, 30-day hospital readmission was statistically greater among patients who initially received inappropriate treatment.

The results of the logistic regression analyses are shown in Tables 4 and 5. Admission from a long-term care facility, antibiotic exposure in the previous 30 days, and COPD were independently associated with the administration of inappropriate treatment. A similar analysis using 30-day hospital readmission as the dependent outcome identified three independent risk factors for this outcome: antibiotic exposure in the previous 30 days, presence of peripheral vascular disease and increasing CURB-65 scores.

\section{Discussion}

This retrospective analysis of patients diagnosed and treated for CAP in the ED reveals that inappropriate antibiotic treatment is common and primarily due to the presence of antibiotic-resistant pathogens including MRSA and non-lactose fermenting Gram-negative bacteria (NLFGNB). We found that almost one half of the patients treated for CAP in the ED had at least one risk factor for healthcare-associated infection. Healthcareassociated risk factors, including prior antibiotic exposure and long-term care residency, were found to be predictors for inappropriate antibiotic treatment. Our study is unique 
Table 1 Baseline characteristics

\begin{tabular}{|c|c|c|c|}
\hline Variable & Appropriate initial therapy $(n=187)$ & Inappropriate initial therapy $(n=72)$ & P value \\
\hline Age, yrs: & $58.1 \pm 17.6$ & $62.0 \pm 15.3$ & 0.103 \\
\hline Male, n (\%): & $109(58.3)$ & $40(55.6)$ & 0.690 \\
\hline \multicolumn{4}{|l|}{ Race, n (\%): } \\
\hline Caucasian & $86(46.0)$ & $31(43.1)$ & \multirow[t]{3}{*}{0.779} \\
\hline African-American & $98(52.4)$ & $40(55.6)$ & \\
\hline Other & $3(1.6)$ & $1(1.4)$ & \\
\hline CURB65 & $1.9 \pm 1.4$ & $2.1 \pm 1.2$ & 0.348 \\
\hline Charlson comorbidity score & $4.5 \pm 3.3$ & $5.4 \pm 1.2$ & 0.090 \\
\hline \multicolumn{4}{|l|}{ Coexisting conditions, n (\%): } \\
\hline Coronary artery disease & $34(18.2)$ & $14(19.4)$ & 0.815 \\
\hline Congestive heart failure & $56(29.9)$ & $31(43.1)$ & 0.045 \\
\hline Peripheral vascular disease & $13(7.0)$ & $13(18.1)$ & 0.008 \\
\hline Cerebral vascular accident & $18(9.6)$ & $10(13.9)$ & 0.322 \\
\hline Dementia & $4(2.1)$ & $0(0.0)$ & 0.578 \\
\hline Chronic obstructive pulmonary disease & $93(49.7)$ & $48(66.7)$ & 0.014 \\
\hline Cirrhosis & $23(12.3)$ & $6(8.3)$ & 0.364 \\
\hline Diabetes & $57(30.5)$ & $24(33.3)$ & 0.657 \\
\hline Chronic kidney disease & $20(10.7)$ & $16(22.2)$ & 0.016 \\
\hline Underlying malignancy & $32(17.1)$ & $12(16.7)$ & 0.932 \\
\hline HIV positive & $1(0.5)$ & $0(0.0)$ & 1.000 \\
\hline \multicolumn{4}{|l|}{ Healthcare-associated risk factors, $n$ (\%): } \\
\hline Hospitalized in preceding 90 days & $36(19.3)$ & $22(30.6)$ & 0.051 \\
\hline Long-term care resident & $2(1.1)$ & $7(9.7)$ & 0.002 \\
\hline Chronic hemodialysis & $3(1.6)$ & $6(8.3)$ & 0.016 \\
\hline Immunocompromised & $38(20.3)$ & $17(23.6)$ & 0.562 \\
\hline Previous antibiotics & $43(23.0)$ & $28(38.9)$ & 0.010 \\
\hline Number of healthcare risk factors: & $0.7 \pm 0.9$ & $1.1 \pm 1.0$ & $<0.001$ \\
\hline Healthcare-associated infection: & $79(42.2)$ & $50(69.4)$ & $<0.001$ \\
\hline ICU admission, n (\%): & $52(27.8)$ & $29(40.3)$ & 0.052 \\
\hline \multicolumn{4}{|l|}{ Respiratory failure, n (\%): } \\
\hline None & $142(75.9)$ & $51(70.8)$ & \multirow[t]{3}{*}{0.630} \\
\hline Intubation/mechanical ventilation & 39 (20.9) & $19(26.4)$ & \\
\hline Mask ventilation & $6(3.2)$ & $2(2.8)$ & \\
\hline Hypotension, n (\%): & $110(58.8)$ & $45(62.5)$ & 0.589 \\
\hline
\end{tabular}

Values are expressed as mean \pm SD. CURB65 = confusion of new onset, urea greater than $7 \mathrm{mmol} / \mathrm{l}$, respiratory rate of 30 breaths per minute or greater, blood pressure less than $90 \mathrm{mmHg}$ systolic or diastolic blood pressure $60 \mathrm{mmHg}$ or less, age 65 or older; HIV = human immunodeficiency virus; ICU = intensive care unit. The Charlson comorbidity score predicts the ten-year mortality for a patient who may have a range of comorbid conditions, such as heart disease, AIDS, or cancer (a total of 22 conditions).

in showing that prior antibiotic exposure, presumably by predisposing to inappropriate antibiotic treatment, may also be associated with subsequent 30-day hospital readmission.

These data add to the growing evidence elucidating the importance of discriminating between patients with pneumonia having healthcare-associated risk factors, including prior antibiotic exposure, from those without such risk factors. In one of the largest studies examining the epidemiology of pneumonia, approximately onethird of all patients with pneumonia admitted to the hospital were due to HCAP [9]. Subsequent studies have also documented that HCAP represents between $30 \%$ and $60 \%$ of pneumonias requiring hospitalization in the US $[11,14,18,19]$, and this trend is also observed in Asia and Europe $[10,12,13,20]$. Our study also confirms that MRSA and NLFGNB are common pathogens in patients 
Table 2 Infection characteristics

\begin{tabular}{|c|c|c|c|}
\hline Variable & Appropriate initial therapy $(n=187)$ & Inappropriate initial therapy $(n=72)$ & $P$ value \\
\hline \multicolumn{4}{|l|}{ Cultures source, $n(\%)$ : } \\
\hline Blood only & $46(24.6)$ & $13(18.1)$ & 0.041 \\
\hline BAL & $5(2.7)$ & $6(8.3)$ & \\
\hline Sputum & $105(56.1)$ & $34(47.2)$ & \\
\hline Tracheal aspirate & $31(16.6)$ & $19(26.4)$ & \\
\hline Achromobacter species & $0(0.0)$ & $1(1.4)$ & 0.278 \\
\hline Acinetobacter species & $1(0.5)$ & $5(6.9)$ & 0.007 \\
\hline Citrobacter species & $1(0.5)$ & $0(0.0)$ & 1.000 \\
\hline Enterobacter species & $1(0.5)$ & $6(8.3)$ & 0.002 \\
\hline Escherichia coli & $11(5.9)$ & $0(0.0)$ & 0.038 \\
\hline Haemophilus influenzae & $36(19.3)$ & $3(4.2)$ & 0.002 \\
\hline Klebsiella pneumoniae & $7(3.7)$ & $1(1.4)$ & 0.450 \\
\hline Legionella species & $1(0.5)$ & $0(0.0)$ & 1.000 \\
\hline Methicillin-resistant Staphylococcus aureus & $0(0.0)$ & $34(47.2)$ & $<0.001$ \\
\hline Methicillin-susceptible Staphylococcus aureus & $36(19.3)$ & $2(2.8)$ & $<0.001$ \\
\hline Moraxella catarrhalis & $5(2.7)$ & $0(0.0)$ & 0.326 \\
\hline Proteus species & $1(0.5)$ & $1(1.4)$ & 0.479 \\
\hline Pseudomonas aeruginosa & $0(0.0)$ & $20(27.8)$ & $<0.001$ \\
\hline Streptococcus pneumoniae & $79(42.2)$ & $3(4.2)$ & $<0.001$ \\
\hline Other Streptococci species & $22(11.8)$ & $3(4.2)$ & 0.097 \\
\hline Polymicrobial, n (\%): & $14(7.5)$ & $5(6.9)$ & 0.881 \\
\hline Positive blood culture, n (\%): & $51(27.3)$ & $17(23.6)$ & 0.549 \\
\hline
\end{tabular}

BAL bronchoalveolar lavage.

presenting to the hospital with pneumonia having HCAP risk factors $[9,13,14]$.

The importance of initial antibiotic therapy in patients admitted to the hospital with pneumonia is suggested by a recent medico-economic analysis conducted by Oster et al. [21]. These investigators attempted to identify risk factors for initial treatment failure in patients with CAP in non-ICU settings, and to characterize the association between initial treatment failure and length of stay, total hospital charges, and mortality. Treatment failure was associated with higher case fatality $(8.5 \%$ vs. $3.3 \%)$, longer hospital stays (mean [SD], 10.1 [8.1] days vs. 4.9 [3.3] days), and higher total hospital charges $(\$ 37,602[\$ 71,876]$ vs. $\$ 14,371[\$ 21,633]$ ) (all comparisons, $\mathrm{p}<0.01)$. An important limitation of their study is that the role of inappropriate initial antibiotic treatment was not examined.

The findings from our study also confirm the recent observation made by Shorr et al. that patients with pneumonia and healthcare-associated risk factors are more likely to require 30-day hospital readmission compared to pneumonia patients without healthcareassociated risk factors [22]. Shorr and coworkers found that admission from a long-term care residency, prior antibiotic administration, recent hospitalization, and immunosuppression were independent predictors for 30 -day readmission. However, our study is unique in focusing on patients diagnosed and treated for presumed CAP in the ED and linking 30-day hospital readmission to the administration of inappropriate

Table 3 Outcomes

\begin{tabular}{lccc}
\hline Outcome & Appropriate initial therapy $(\mathbf{n = 1 8 7})$ & Inappropriate initial therapy $(\mathbf{n}=\mathbf{7 2})$ & $\mathbf{P}$ value \\
\hline Hospital mortality, $\mathrm{n}(\%):$ & $13(7.0)$ & $6(8.3)$ & $10.3 \pm 12.0$ \\
Hospital length of stay, days*: & $7.0 \pm 8.9$ & $6.95[4.0,12.45]$ \\
& $4.0[3.0,8.0]$ & $17(23.6)$ & 0.017 \\
30-day hospital readmission, $\mathrm{n}(\%):$ & $23(12.3)$ & 0.024 \\
\hline
\end{tabular}

*Data expressed as mean \pm SD and median with $25^{\text {th }}$ and $75^{\text {th }}$ percentiles. 
Table 4 Multivariate analyses of independent risk factors for inappropriate initial antibiotic treatment*

\begin{tabular}{lccc}
\hline Variable & Adjusted odds ratio & $\mathbf{9 5 \%} \mathbf{C l}$ & P value \\
\hline Long-term care resident & 9.05 & $3.93-20.84$ & 0.008 \\
Antibiotic exposure in previous 30 days & 1.85 & $1.35-2.52$ & 0.049 \\
COPD & 2.05 & $1.52-2.78$ & 0.018 \\
\hline
\end{tabular}

*Other covariates not in the table had a $\mathrm{p}$ value $>0.05$ including previous hospitalization, mechanical ventilation or mask ventilation, CURB65 score, Charlson score, congestive heart failure, peripheral vascular disease, chronic kidney disease, hemodialysis, and ICU admission (Hosmer-Lemeshow goodness-of-fit test, $\mathrm{p}=0.726)$. ICU = intensive care unit; COPD = chronic obstructive pulmonary disease, CURB65 = confusion of new onset, urea greater than 7 mmol/l, respiratory rate of 30 breaths per minute or greater, blood pressure less than $90 \mathrm{mmHg}$ systolic or diastolic blood pressure $60 \mathrm{mmHg}$ or less, age 65 or older.

antibiotic treatment, which in turn was independently associated with prior antibiotic exposure. Prior antibiotic exposure has been shown to be an important risk factor for the development of certain types of pneumonia to include drug-resistant Streptococcus pneumoniae [23], ventilator-associated pneumonia [24] and HCAP [13]. The common link among these types of pneumonia is infection due to antibiotic-resistant bacteria. The presence of a nexus between physicianprescribed antibiotic therapy (e.g., prior antibiotic exposure, inappropriate antibiotic treatment) and hospital readmission underscores that modifiable patient characteristics may play a role as key determinants of whether a hospitalized patient with pneumonia eventually requires readmission.

There are several important limitations of our study that should be noted. First, the study was performed at an academic medical center and the results may not be generalizable to other institutions, especially those with a lower incidence of MDR pathogens. The large proportion of pneumonia patients with HCAP risk factors receiving broad-spectrum antibiotic therapy at our institution underscores this point. However, our findings are in agreement with those from other investigators supporting their overall validity [9-16,20]. Second, we did not examine all aspects of medical care that may have influenced the prescription of inappropriate therapy and the outcomes of our patient cohort. For example, lack of physician experience or knowledge regarding the importance of broader empiric antibiotic coverage for patients with pneumonia and healthcare-associated risk factors could have contributed to our results [25]. Third, the retrospective nature of this study limits our ability to infer causality between the identified risk factors and inappropriate antibiotic therapy. Fourth, we did not identify the specific causes or indications for the 30-day readmissions. It is possible that the cause of readmission in many cases may not have been related to the prior episode of pneumonia or inappropriate treatment of the pneumonia. Our analysis found that the presence of peripheral vascular disease, greater CURB-65 scores, and prior antibiotic exposure predispose to readmission. These could all be considered to be markers of greater co-morbidities or severity of illness accounting for the readmissions.

Another important limitation of our study is that not all healthcare-associated risk factors carry the same importance. A US study found recent hospitalization, residency in a long-term care facility, chronic hemodialysis, and admission to the ICU to be the best predictors of pneumonia due to an antibiotic-resistant pathogen [26]. These findings are similar to those from a European study that identified prior hospitalization, residence in a long-term care setting, and chronic renal failure as predictors of antibiotic resistance in pneumonia [27]. However, a study from South Korea found prior antibiotic exposure and recent hospitalization to be independent predictors for antibiotic resistance [28]. Similarly, not all investigators have found antibiotic resistance and inappropriate therapy to be problematic in patients with pneumonia and healthcare-associated risk factors [29,30]. Several recent studies suggest adverse outcomes with broader spectrum therapy of pneumonia [31,32]. This has resulted in a call to avoid the use of the term HCAP and instead to refer to certain patients with pneumonia as being at increased risk for antibiotic-resistance due to their specific risk profile [33]. Moreover, it is important to acknowledge that our study only included culture-positive

Table 5 Multivariate analyses of independent risk factors for 30-day hospital readmission*

\begin{tabular}{lccc}
\hline Variable & Adjusted odds ratio & 95\% Cl & P value \\
\hline Antibiotic exposure in previous 30 days & 2.12 & $1.45-3.10$ & 0.047 \\
Peripheral vascular disease & 3.64 & $2.27-5.84$ & 0.006 \\
CURB 65 (1-point increments) & 1.46 & $1.27-1.68$ & 0.007
\end{tabular}

*Other covariates not in the table had a $\mathrm{p}$ value $>0.05$ including inappropriate initial antibiotic treatment, age, chronic dialysis, intensive care unit admission, COPD, congestive heart failure, and the Charlson score (Hosmer-Lemeshow goodness-of-fit test, $p=0.431$ ). ICU = intensive care unit; COPD $=$ chronic obstructive pulmonary disease, CURB65 = confusion of new onset, urea greater than $7 \mathrm{mmol} / \mathrm{l}$, respiratory rate of 30 breaths per minute or greater, blood pressure less than $90 \mathrm{mmHg}$ systolic or diastolic blood pressure $60 \mathrm{mmHg}$ or less, age 65 or older. 
patients. Therefore, our data represent the more severe end of the CAP/HCAP spectrum. The use of empiric HCAP regimens do not appear to be justified in culturenegative patients with less severe disease [34].

\section{Conclusions}

In conclusion we found that inappropriate therapy for patients diagnosed and treated for CAP in the ED setting is relatively common. Patients receiving inappropriate therapy were significantly more likely to have HCAP risk factors compared to patients receiving appropriate therapy, suggesting that the identification of these risk factors, or recognition of their clinical importance, was underappreciated in these individuals. The association of inappropriate therapy with longer hospital lengths of stay and greater 30-day hospital readmission suggests that efforts to reduce inappropriate therapy in the ED would be beneficial. Several investigations have applied multi-disciplinary approaches to the management of such patients demonstrating improvements in antibiotic prescription to include more targeted use of antimicrobial agents [35,36]. Future studies evaluating rapid microbiologic techniques, informatics systems for the timely identification of prior antibiotic exposure, and utility of new antimicrobial agents are needed to improve the treatment strategies for patients with pneumonia [37]. In the interim, clinician identification of risk factors for antimicrobial resistance should be emphasized in patients presenting to the hospital with pneumonia in order to optimize the balance between providing appropriate therapy and avoiding unnecessary antibiotic exposure [38].

\begin{abstract}
Abbreviations
AOR: Adjusted odds ratio; ATS/IDSA: American Thoracic Society/Infectious Disease Society of America; CAP: Community-acquired pneumonia;

$\mathrm{Cl}$ : Confidence interval; COPD: Chronic obstructive pulmonary disease; ED: Emergency department; HAP: Hospital-acquired pneumonia; HCAP: Healthcare-associated pneumonia; HIV: Human immunodeficiency virus; ICU: Intensive care unit; MDR: Multidrug-resistant; MRSA: Methicillinresistant Staphylococcus aureus; MSSA: Methicillin-susceptible Staphylococcus
\end{abstract} aureus; NLFGNB: Non-lactose fermenting Gram-negative bacteria.

\section{Competing interests}

Kollef has served as a consultant, speaker for, or received grant support from: Cubist, Hospria, Merck, and Cardeas.

Dr. Micek has received grant support from Cubist, Forest, Optimer, Merck, and Pfizer.

The remaining authors have no potential conflicts.

\section{Authors' contributions}

MHK, STM, NBH: had full access to all of the data in the study and take responsibility for the integrity of the data and the accuracy of the data analysis; contributed to the study conception and design, statistical analysis, drafting of the manuscript, and have given approval to the final version. AL: had full access to all of the data in the study and takes responsibility for the integrity of the data and the accuracy of the data analysis; contributed to the study conception and data base construction, and drafting of the manuscript and has given approval to the final version. BMF: had full access to all of the data in the study and takes responsibility for the integrity of the data and the accuracy of the data analysis; contributed to the study conception and design and drafting of the manuscript and has given approval to the final version. All authors read and approved the final manuscript.

\section{Funding source}

This work was supported by an unrestricted grant from Forest Pharmaceuticals. Dr. Kollef's effort was supported by the Barnes-Jewish Hospital Foundation. Dr. Fuller's effort was supported by the National Center for Research Resources (NCRR) and the National Center for Advancing Translational Sciences (NCATS), National Institutes of Health $(\mathrm{NIH})$, through Grant Number UL1 TR 000448 .

\section{Role of sponsors}

This research was supported by an unrestricted grant from Forest Pharmaceuticals. The sponsor (Forest Pharmaceuticals) had no role in any aspect of this investigation to include study conception and design, statistical analysis, and critical revision of the manuscript.

\section{Author details}

${ }^{1}$ St. Louis College of Pharmacy, 4588 Parkview Place, St. Louis, MO 63110-1088, USA. ${ }^{2}$ Creighton University, 2500 California Plaza, Omaha, NE 68178 , USA. ${ }^{3}$ Washington University School of Medicine, 660 South Euclid Avenue, Campus Box 8072, St. Louis, MO 63110, USA. ${ }^{4}$ BJC Learning Institute, 8300 Eager Road, Mail Stop 92-92-241, St. Louis, MO 63144, USA. ${ }^{5}$ Division of Pulmonary and Critical Care Medicine, Washington University School of Medicine, 660 South Euclid Avenue, Campus Box 8052, St. Louis, MO 63110, USA.

Received: 31 October 2013 Accepted: 4 February 2014

Published: 5 February 2014

\section{References}

1. Mandell LA, Wunderink RG, Anzueto A, Bartlett JG, Campbell GD, Dean NC, Dowell SF, File TM Jr, Musher DM, Niederman MS, Torres A, Whitney CG, Infectious Diseases Society of America; American Thoracic Society: Infectious Diseases Society of America/American Thoracic Society consensus guidelines on the management of community-acquired pneumonia in adults. Clin Infect Dis 2007, 44:S27-S72.

2. Welte T, Torres A, Nathwani D: Clinical and economic burden of community-acquired pneumonia among adults in Europe. Thorax 2012, 67:71-79.

3. File TM Jr, Marrie TJ: Burden of community-acquired pneumonia in North American adults. Postgrad Med 2010, 122:130-141.

4. Huang SS, Johnson KM, Ray GT, Wroe P, Lieu TA, Moore MR, Zell ER, Linder JA, Grijalva CG, Metlay JP, Finkelstein JA: Healthcare utilization and cost of pneumococcal disease in the United States. Vaccine 2011, 29:3398-3412.

5. Ewig S, Torres A: Community-acquired pneumonia as an emergency: time for an aggressive intervention to lower mortality. Eur Respir J 2011, 38:253-260

6. Feldman C, Anderson R: Antibiotic resistance of pathogens causing community-acquired pneumonia. Semin Respir Crit Med 2012, 33:232-243.

7. Fujitani S, Yu VL: A new category-healthcare-associated pneumonia: a good idea, but problems with its execution. Eur J Clin Microbiol Infect Dis 2006, 25:627-631.

8. American Thoracic Society; Infectious Diseases Society of America: Guidelines for the management of adults with hospital-acquired, ventilator-associated, and healthcare-associated pneumonia. Am J Respir Crit Care Med 2005, 71:388-416.

9. Kollef MH, Shorr A, Tabak YP, Gupta V, Liu LZ, Johannes RS: Epidemiology and outcomes of health-care-associated pneumonia: results from a large US database of culture-positive pneumonia. Chest 2005, 128:3854-3862.

10. Venditti M, Falcone M, Corrao S, Licata G, Serra P, Study Group of the Italian Society of Internal Medicine: Outcomes of patients hospitalized with community-acquired, healthcare- associated, and hospital-acquired pneumonia. Ann Intern Med 2009, 150:19-26.

11. Hsu JL, Siroka AM, Smith MW, Holodniy M, Meduri GU: One-year outcomes of community-acquired and healthcare-associated pneumonia in the Veterans Affairs Healthcare System. Int J Infect Dis 2011, 15:e382-e387.

12. Jung JY, Park MS, Kim YS, Park BH, Kim SK, Chang J, Kang YA: Healthcareassociated pneumonia among hospitalized patients in a Korean tertiary hospital. BMC Infect Dis 2011, 11:61. 
13. Falcone $M$, Venditti $M$, Shindo $Y$, Kollef MH: Healthcare-associated pneumonia: diagnostic criteria and distinction from community-acquired pneumonia. Int J Infect Dis 2011, 15:e545-e550.

14. Micek ST, Kollef KE, Reichley RM, Roubinian N, Kollef MH: Health careassociated pneumonia and community-acquired pneumonia: a singlecenter experience. Antimicrob Agents Chemother 2007, 51:3568-3573.

15. Kollef MH, Morrow LE, Baughman RP, Craven DE, McGowan JE Jr, Micek ST, Niederman MS, Ost D, Paterson DL, Segreti J: Health care-associated pneumonia (HCAP): a critical appraisal to improve identification, management, and outcomes-proceedings of the HCAP Summit. Clin Infect Dis 2008, 46:S296-S334.

16. Micek ST, Reichley RM, Kollef MH: Health care-associated pneumonia (HCAP): empiric antibiotics targeting methicillin-resistant Staphylococcus aureus (MRSA) and Pseudomonas aeruginosa predict optimal outcome. Medicine (Baltimore) 2011, 90:390-395.

17. Lim WS, van der Eerden MM, Laing R, Boersma WG, Karalus N, Town Gl, Lewis SA, Macfarlane JT: Defining community acquired pneumonia severity on presentation to hospital: an international derivation and validation study. Thorax 2003, 58:377-382.

18. Shorr AF, Zilberberg MD, Micek ST, Kollef MH: Prediction of infection due to antibiotic resistant bacteria by select risk factors for health careassociated pneumonia. Arch Intern Med 2008, 168:2205-2210.

19. Schreiber MP, Chan CM, Shorr AF: Resistant pathogens in nonnosocomial pneumonia and respiratory failure: is it time to refine the definition of health-care-associated pneumonia? Chest 2010, 137:1283-1288.

20. Shindo Y, Sato S, Maruyama E, Ohashi T, Ogawa M, Hashimoto N, Imaizumi K, Sato T, Hasegawa Y: Health-care-associated pneumonia among hospitalized patients in a Japanese community hospital. Chest 2009, 135:633-640.

21. Oster G, Berger A, Edelsberg J, Weber DJ: Initial treatment failure in non-ICU community-acquired pneumonia: risk factors and association with length of stay, total hospital charges, and mortality. J Med Econ 2013, 16:809-819.

22. Shorr AF, Zilberberg MD, Reichley R, Kan J, Hoban A, Hoffman J, Micek ST, Kollef $\mathrm{MH}$ : Readmission following hospitalization for pneumonia: the impact of pneumonia type and its implication for hospitals. Clin Infect Dis 2013, 57:362-367.

23. Jenkins TC, Sakai J, Knepper BC, Swartwood CJ, Haukoos JS, Long JA, Price CS, Burman WJ: Risk factors for drug-resistant Streptococcus pneumoniae and antibiotic prescribing practices in outpatient community-acquired pneumonia. Acad Emerg Med 2012, 19:703-706.

24. Kollef $\mathrm{MH}$ : Ventilator-associated pneumonia. A multivariate analysis. JAMA 1993, 270:1965-1970.

25. Seymann GB, Di Francesco L, Sharpe B, Rohde J, Fedullo P, Schneir A, Fee C, Chan KM, Fatehi P, Dam TT: The HCAP gap: differences between self-reported practice patterns and published guidelines for health care-associated pneumonia. Clin Infect Dis 2009, 49:1868-1874

26. Shorr AF, Zilberberg MD, Reichley R, Kan J, Hoban A, Hoffman J, Micek ST, Kollef $\mathrm{MH}$ : Validation of a clinical score for assessing the risk of resistant pathogens in patients with pneumonia presenting to the emergency department. Clin Infect Dis 2012, 54:193-198.

27. Aliberti S, Di Pasquale M, Zanaboni AM, Cosentini R, Brambilla AM, Seghezzi S, Tarsia P, Mantero M, Blasi F: Stratifying risk factors for multidrug-resistant pathogens in hospitalized patients coming from the community with pneumonia. Clin Infect Dis 2012, 54:470-478.

28. Park SC, Kang YA, Park BH, Kim EY, Park MS, Kim YS, Kim SK, Chang J, Jung JY: Poor prediction of potentially drug-resistant pathogens using current criteria of health care-associated pneumonia. Respir Med 2012, 106:1311-1319.

29. Garcia-Vidal C, Viasus D, Roset A, Adamuz J, Verdaguer R, Dorca J, Gudiol F, Carratalà J: Low incidence of multidrug-resistant organisms in patients with healthcare-associated pneumonia requiring hospitalization. Clin Microbiol Infect 2011, 17:1659-1665.

30. Chalmers JD, Taylor JK, Singanayagam A, Fleming GB, Akram AR, Mandal P, Choudhury G, Hill AT: Epidemiology, antibiotic therapy, and clinical outcomes in health care-associated pneumonia: a UK cohort study. Clin Infect Dis 2011, 53:107-113.

31. Kett DH, Cano E, Quartin AA, Mangino JE, Zervos MJ, Peyrani P, Cely CM, Ford KD, Scerpella EG, Ramirez JA, Improving Medicine through Pathway Assessment of Critical Therapy of Hospital-Acquired Pneumonia (IMPACT-HAP) Investigators: Implementation of guidelines for management of possible multidrug-resistant pneumonia in intensive care: an observational, multicentre cohort study. Lancet Infect Dis 2011, 11:181-189.

32. Attridge RT, Frei CR, Restrepo MI, Lawson KA, Ryan L, Pugh MJ, Anzueto A, Mortensen EM: Guideline-concordant therapy and outcomes in healthcare-associated pneumonia. Eur Respir J 2011, 38:878-887.

33. Ewig S, Welte T, Torres A: Is healthcare-associated pneumonia a distinct entity needing specific therapy? Curr Opin Infect Dis 2012, 25:166-175.

34. Labelle AJ, Arnold H, Reichley RM, Micek ST, Kollef MH: A comparison of culture-positive and culture-negative health-care-associated pneumonia. Chest 2010, 137:1130-1137.

35. Taylor SP, Taylor BT: Healthcare-associated pneumonia in hemodialysis patients: Clinical outcomes in patients treated with narrow versus broad spectrum antibiotic therapy. Respirology 2013, 18:364-367.

36. Falcone M, Corrao S, Licata G, Serra P, Venditti M: Clinical impact of broadspectrum empirical antibiotic therapy in patients with healthcare-associated pneumonia: a multicenter interventional study. Intern Emerg Med 2012, 7:523-531.

37. Lawrence $\mathrm{KL}$, Kollef MH: Antimicrobial stewardship in the intensive care unit: advances and obstacles. Am J Respir Crit Care Med 2009, 179:434-438.

38. Shindo $Y$, Ito R, Kobayashi D, Ando M, Ichikawa M, Shiraki A, Goto Y, Fukui Y, Iwaki M, Okumura J, Yamaguchi I, Yagi T, Tanikawa Y, Sugino Y, Shindoh J, Ogasawara T, Nomura F, Saka H, Yamamoto M, Taniguchi H, Suzuki R, Saito H, Kawamura T, Hasegawa Y: Risk factors for drug-resistant pathogens in community-acquired and healthcare-associated pneumonia. Am J Respir Crit Care Med 2013, 188:985-995.

doi:10.1186/1471-2334-14-61

Cite this article as: Micek et al:: Clinical implications for patients treated inappropriately for community-acquired pneumonia in the emergency department. BMC Infectious Diseases 2014 14:61.

\section{Submit your next manuscript to BioMed Central and take full advantage of:}

- Convenient online submission

- Thorough peer review

- No space constraints or color figure charges

- Immediate publication on acceptance

- Inclusion in PubMed, CAS, Scopus and Google Scholar

- Research which is freely available for redistribution 